\title{
Optimal and Simultaneous Designs of Hermitian Transforms and Masks for Reducing Intraclass Separations of Feature Vectors for Anomaly Detection of Diabetic Retinopathy Images
}

\author{
${ }^{1}$ S. R. Subramaniam, ${ }^{1}$ A. Georgakis \\ ${ }^{1}$ Dept. of Elec. Eng., \\ King's College London, \\ London, WC2R 2LS, U.K. \\ suba.r.subramaniam@kcl.ac.uk \\ apostolos.georgakis@kcl.ac.uk
}

\author{
${ }^{2}$ B. W. K. Ling \\ ${ }^{2}$ School of Engineering, \\ University of Lincoln, \\ Lincoln, LN6 7TS, U.K. \\ wling@lincoln.ac.uk
}

\author{
${ }^{3}$ J. Goh, ${ }^{3}$ H. L. Tang \\ ${ }^{3}$ Dept. of Computing, \\ University of Surrey, \\ Guildford, Surrey, GU2 7XH, U.K. \\ jonathan.goh@surrey.ac.uk \\ h.tang@surrey.ac.uk
}

${ }^{3,4}$ T. Peto, ${ }^{5}$ G. Saleh

${ }^{4}$ NIHR Biomedical Research Centre for Ophthalmology, at Moorfields Eye Hospital NHS Foundation Trust and UCL Institute of Ophthalmology EC1V 2PD, UK, Tunde.Peto@moorfields.nhs.uk ${ }^{5}$ Moorfields Eye Hospital, MK42 9DJ, UK, George.Saleh@moorfields.nhs.uk

\begin{abstract}
This paper proposes a novel methodology for the optimal and simultaneous designs of both Hermitian transforms and masks for reducing the intraclass separations of feature vectors for anomaly detection of diabetic retinopathy images. Each class of training images associates with a Hermitian transform, a mask and a known represented feature vector. The optimal and simultaneous designs of both the Hermitian transforms and the masks are formulated as least squares optimization problems subject to the Hermitian constraints. Since the optimal mask of each class of training images is dependent on the corresponding optimal Hermitian transform, only the Hermitian transforms are required to be designed. Nevertheless, the Hermitian transform design problems are optimization problems with highly nonlinear objective functions subject to the complex valued quadratic Hermitian constraints. This kind of optimization problems is very difficult to solve. To address the difficulty, this paper proposes a singular value decomposition approach for deriving a condition on the solutions of the optimization problems as well as an iterative approach for solving the optimization problems. Since the matrices characterizing the discrete Fourier transform, discrete cosine transform and discrete fractional Fourier transform are Hermitian, the Hermitian transforms designed by our proposed approach are more general than existing transforms. After both the Hermitian transforms and the masks for all classes of
\end{abstract}

training images are designed, they are applied to test images. The test images will assign to the classes where the Euclidean 2norms of the differences between the processed feature vectors of the test images and the corresponding represented feature vectors are minimum. Computer numerical simulation results show that the proposed methodology for the optimal and simultaneous designs of both the Hermitian transforms and the masks is very efficient and effective. The proposed technique is also very efficient and effective for reducing the intraclass separations of feature vectors for anomaly detection of diabetic retinopathy images.

\section{INTRODUCTION}

A common complication of diabetes is diabetic retinopathy (DR), an eye disease that is one of the major causes of blindness for adults under the age of 65. Diabetic retinopathy is a progressive condition, diagnosing it at early stages provides time to treat the disease and to allow for maintenance of good vision. In the UK, this is done through a national screening programme. There are about 2.8 million patients in the UK be screened yearly, with the requirement that there are least 4 gradable fundus pictures to be taken for each patient. Of all screening images, about $2 / 3 \mathrm{rd}$ is normal. On average, 
graders take much longer time to look at normal images than deem someone abnormal. This motivates the research of developing an efficient and automatic system to assist DR image analysis or screening. Previous work in DR image analysis mostly requires the use of sophisticated algorithms [1]-[4] to locate abnormal DR signs in order to judge the abnormality of an image. However, when the goal of an automated system is to filter out the majority of normal images where most of the processed images are normal, such strategy usually has too low specificity to be effective. Our current system, implemented through complex evolutionary approach [5], although proved to have high sensitivity and specificity, it requires very expensive computational time. While looking at the other side of the problem, normal fundus images have their own inherent characteristics that have not been fully explored. This research investigates the separation of normal and abnormal images solely based on global characteristics of these two types of images.

In order to have a fast and efficient screening process, only few features can be used for screening. However, when the dimension of the feature vectors is small, the feature vectors will be nonlinearly separable [6] in the feature space. To tackle this difficulty, the most common method is to apply a transform [7], such as the discrete Fourier transform, discrete cosine transform or discrete fractional Fourier transform [8], as well as a mask and the corresponding inverse transform to the feature vectors, so that the intraclass separations of the processed feature vectors is small. By applying such technique, it can significantly improve the recognition performances.

However, there are some fundamental unaddressed issues. First, although there are some common transforms, such as the discrete Fourier transform, discrete cosine transform and discrete fractional Fourier transform, it is not guaranteed that one of them is optimal. If this is not the case, then it requires to design the optimal transform. Nevertheless, this issue has not been addressed yet. Second, as the mask is mutually dependent on the transform, it requires to determine both the optimal transform and the mask simultaneously. Similar, this issue has also not been addressed yet. Third, but not the last, as all the existing transforms are Hermitian, it is useful to design an optimal Hermitian transform. However, by resolving the relationship between the optimal mask and the optimal Hermitian transform, the objective function will be highly nonlinear. As the Hermitian constraints are complex valued quadratic constraints, the optimal designs of the Hermitian transforms become highly nonlinear complex valued quadratic constrained optimization problems. Solving such kind of optimization problems has not been addressed yet [9].

To tackle these difficulties, this paper proposes a singular value decomposition approach to derive a condition on the optimal solutions of the optimization problems, as well as an iterative approach for solving the optimization problems. The outline of this paper is as follows. Notations used throughout this paper and the problem formulation are presented in Section II. The solution method for solving the optimization problems is presented in Section III. Computer numerical simulation results are presented in Section IV. Finally, a conclusion is drawn in Section V.

\section{NOTATIONS AND PROBLEM FormULATION}

Assume that there are $C$ different classes of images. For each class of images, assume that there are $M_{c}$ training images for $c=0,1, \cdots, C-1$. Also, assume that features have been extracted for each image and the feature vectors are in the $N$-dimensional real valued Euclidean space denoted as

$$
\mathbf{x}_{c, i} \equiv\left[\begin{array}{lll}
x_{c, i, 0} & \cdots & x_{c, i, N-1}
\end{array}\right]^{T} \in \mathfrak{R}^{N \times 1}
$$

for $i=0,1, \cdots, M_{c}-1$ and for $c=0,1, \cdots, C-1$. Here, $\mathfrak{R}^{a \times b}$ denotes the space of $a \times b$ real valued matrices. For each class of images, assume that there is a represented feature vector. Let

$$
\mathbf{y}_{c} \equiv\left[\begin{array}{lll}
y_{c, 0} & \cdots & y_{c, N-1}
\end{array}\right]^{T} \in \mathfrak{R}^{N \times 1} \text { for } c=0,1, \cdots, C-1
$$

be the represented feature vector in the $c^{\text {th }}$ class. Suppose that $\mathbf{y}_{c}$ for $c=0,1, \cdots, C-1$ are known.

Denote $C^{a \times b}$ as the space of $a \times b$ complex valued matrices. Let $\mathbf{U}_{c} \in C^{N \times N}$ for $c=0,1, \cdots, C-1$ be a Hermitian transform corresponding to the $c^{\text {th }}$ class. That is,

$$
\mathbf{U}_{c} \mathbf{U}_{c}^{H}=\mathbf{U}_{c}^{H} \mathbf{U}_{c}=\mathbf{I}_{N \times N} \text { for } c=0,1, \cdots, C-1,
$$

where the superscript " $H$ " represents the Hermitian operator or the conjugate transpose operator and $\mathbf{I}_{N \times N}$ denotes the $N \times N$ identity matrix. Let

$$
\mathbf{f}_{c} \equiv\left[\begin{array}{lll}
f_{c, 0} & \cdots & f_{c, N-1}
\end{array}\right]^{T} \in \mathfrak{R}^{N \times 1} \text { for } c=0,1, \cdots, C-1
$$

be the vector of the mask coefficients for the $c^{\text {th }}$ class. Our objective is to optimally and simultaneously design both the Hermitian transforms and the masks so that the intraclass separations of the processed feature vectors are as small as possible. Here, the smallness is defined based on the least squares criterion because this is the most common criterion used to measure the closeness of the vectors in any vector spaces. Hence, this paper also employs the least squares criterion as the objective function. Let $\operatorname{diag}(\mathbf{z})$ be a diagonal matrix with its diagonal elements being the elements of the vector $\mathbf{z}$. Define

$$
\mathbf{F}_{c} \equiv \operatorname{diag}\left(\mathbf{f}_{c}\right) \text { for } c=0,1, \cdots, C-1 .
$$

Then, the optimal and simultaneous design of both the Hermitian transform and the mask function for the $c^{\text {th }}$ class of images can be formulated as the following optimization problem:

$$
\min _{\left(\mathbf{U}_{c}, \mathbf{f}_{c}\right)} \quad J\left(\mathbf{U}_{c}, \mathbf{f}_{c}\right) \equiv \sum_{i=0}^{M_{c}-1}\left\|\mathbf{U}_{c}^{H} \mathbf{F}_{c} \mathbf{U}_{c} \mathbf{x}_{c, i}-\mathbf{y}_{c}\right\|^{2},
$$

subject to $\mathbf{U}_{c} \mathbf{U}_{c}^{H}=\mathbf{U}_{c}^{H} \mathbf{U}_{c}=\mathbf{I}_{N \times N}$,

where $\|\cdot\|$ represents the Euclidean 2-norm operator.

For a given $\mathbf{U}_{c}$, where $c=0,1, \cdots, C-1$, let the optimal mask be 


$$
\mathbf{f}_{c}^{\bullet} \equiv\left[\begin{array}{lll}
f_{c, 0}^{\bullet} & \cdots & f_{c, N-1}^{\bullet}
\end{array}\right]^{T} \text { for } c=0,1, \cdots, C-1 .
$$

As the problem involves the optimal and simultaneous design of both $\mathbf{U}_{c}$ and $\mathbf{f}_{c}$ for $c=0,1, \cdots, C-1, \mathbf{f}_{c}^{\bullet}$ depends on $\mathbf{U}_{c}$ for $c=0,1, \cdots, C-1$. Let $\operatorname{Re}(\mathbf{z})$ be the real part of the matrix $\mathbf{z}$ and $\widetilde{\mathbf{I}}_{n, n} \in \mathfrak{R}^{N \times N}$ be the matrix with all the elements being zero except the element in the $(n+1)^{\text {th }}$ row and the $(n+1)^{t h}$ column being one. Then, the following theorem analytically solves $\mathbf{f}_{c}^{\bullet}$ for a given $\mathbf{U}_{c}$, where $c=0,1, \cdots, C-1$.

\section{Theorem 1}

$$
f_{c, n}^{\bullet}=\frac{\sum_{i=0}^{M_{c}-1} \operatorname{Re}\left(\mathbf{y}_{c}^{T} \mathbf{U}_{c}^{H} \widetilde{\mathbf{I}}_{n, n} \mathbf{U}_{c} \mathbf{x}_{c, i}\right)}{\sum_{i=0}^{M_{c}-1} \mathbf{x}_{c, i}^{T} \mathbf{U}_{c}^{H} \widetilde{\mathbf{I}}_{n, n} \mathbf{U}_{c} \mathbf{x}_{c, i}}
$$

for $c=0,1, \cdots, C-1$ and for $n=0,1, \cdots, N-1$.

For a given $\mathbf{U}_{c}$, where $c=0,1, \cdots, C-1, \mathbf{f}_{c}^{\bullet}$ for $c=0,1, \cdots, C-1$ can be derived based on the result stated in Theorem 1. Hence, the optimal and simultaneous design problem of both $\mathbf{U}_{c}$ and $\mathbf{f}_{c}$ for $c=0,1, \cdots, C-1$ now becomes the optimal design problem of $\mathbf{U}_{c}$ for $c=0,1, \cdots, C-1$ only. Let

$$
\hat{\mathbf{X}}_{c} \equiv\left[\begin{array}{ccc}
\sum_{i=0}^{M_{c}-1} x_{c, i, 0} x_{c, i, 0} & \cdots & \sum_{i=0}^{M_{c}-1} x_{c, i, 0} x_{c, i, N-1} \\
\vdots & \ddots & \vdots \\
\sum_{i=0}^{M_{c}-1} x_{c, i, N-1} x_{c, i, 0} & \cdots & \sum_{i=0}^{M_{c}-1} x_{c, i, N-1} x_{c, i, N-1}
\end{array}\right]
$$

for $c=0,1, \cdots, C-1$,

$$
\hat{\mathbf{Y}}_{c} \equiv\left[\begin{array}{ccc}
\sum_{i=0}^{M_{c}-1} y_{c, 0} x_{c, i, 0} & \cdots & \sum_{i=0}^{M_{c}-1} y_{c, 0} x_{c, i, N-1} \\
\vdots & \ddots & \vdots \\
\sum_{i=0}^{M_{c}-1} y_{c, N-1} x_{c, i, 0} & \cdots & \sum_{i=0}^{M_{c}-1} y_{c, N-1} x_{c, i, N-1}
\end{array}\right]
$$

for $c=0,1, \cdots, C-1$, and

$$
\mathbf{U}_{c} \equiv\left[\begin{array}{c}
\mathbf{u}_{c, 0}^{T} \\
\vdots \\
\mathbf{u}_{c, N-1}^{T}
\end{array}\right] \text { for } c=0,1, \cdots, C-1 .
$$

Then, the following theorem reformulates the original optimal and simultaneous design problem of both $\mathbf{U}_{c}$ and $\mathbf{f}_{c}$ for $c=0,1, \cdots, C-1$ to the optimal design problem of $\mathbf{U}_{c}$ for $c=0,1, \cdots, C-1$.

Theorem 2
The original optimal and simultaneous design problem of both $\mathbf{U}_{c}$ and $\mathbf{f}_{c}$ for $c=0,1, \cdots, C-1$ is equivalent to the following optimal design problem of $\mathbf{U}_{c}$ for $c=0,1, \cdots, C-1$ :

$$
\min _{\mathbf{U}_{c}} \quad \widetilde{J}\left(\mathbf{U}_{c}\right) \equiv M_{c} \mathbf{y}_{c}^{T} \mathbf{y}_{c}-\sum_{n=0}^{N-1} \frac{\left(\operatorname{Re}\left(\mathbf{u}_{c, n}^{H} \hat{\mathbf{Y}}_{c} \mathbf{u}_{c, n}\right)\right)^{2}}{\mathbf{u}_{c, n}^{H} \hat{\mathbf{X}}_{c} \mathbf{u}_{c, n}},
$$

subject to $\mathbf{U}_{c} \mathbf{U}_{c}^{H}=\mathbf{U}_{c}^{H} \mathbf{U}_{c}=\mathbf{I}_{N \times N}$.

By Theorem 2, we only need to optimize $\mathbf{U}_{c}$ for $c=0,1, \cdots, C-1$. After we obtain the optimal $\mathbf{U}_{c}$ for $c=0,1, \cdots, C-1, \mathbf{f}_{c}^{\bullet}$ for $c=0,1, \cdots, C-1$ can be derived based on Theorem 1. However, the optimization problem consists of complex valued quadratic constraints. This kind of optimization problems is very difficult to solve. To tackle this difficulty, a Lagrange approach is proposed to convert the constrained optimization problem to an unconstrained optimization problem. Let $\lambda_{c, p, q}$ for $c=0,1, \cdots, C-1$, for $p=0,1, \cdots, N-1$ and for $q=0,1, \cdots, N-1$ be the Lagrange multipliers of the optimization problem,

$$
\lambda_{c} \equiv\left[\begin{array}{ccc}
\lambda_{c, 0,0} & \cdots & \lambda_{c, 0, N-1} \\
\vdots & \ddots & \vdots \\
\lambda_{c, N-1,0} & \cdots & \lambda_{c, N-1, N-1}
\end{array}\right] \text { for } c=0,1, \cdots, C-1
$$

be the matrices containing these Lagrange multipliers, and $\delta($.$) be the discrete time delta function. Then, the constrained$ optimization problem is equivalent to the following unconstrained optimization problem:

$$
\begin{aligned}
\min _{\left(\mathbf{U}_{c}, \boldsymbol{\lambda}_{c}\right)} \hat{J}\left(\mathbf{U}_{c}, \boldsymbol{\lambda}_{c}\right) \equiv & M_{c} \mathbf{y}_{c}^{T} \mathbf{y}_{c}-\sum_{n=0}^{N-1} \frac{\left(\operatorname{Re}\left(\mathbf{u}_{c, n}^{H} \hat{\mathbf{Y}}_{c} \mathbf{u}_{c, n}\right)\right)^{2}}{\mathbf{u}_{c, n}^{H} \hat{\mathbf{X}}_{c} \mathbf{u}_{c, n}} \\
& -\sum_{p=0}^{N-1} \sum_{q=0}^{N-1} \lambda_{c, p, q}\left(\mathbf{u}_{c, p}^{H} \mathbf{u}_{c, q}-\delta(p-q)\right)
\end{aligned}
$$

for $c=0,1, \cdots, C-1$. However, the objective function of this unconstrained optimization problem is high nonlinear. This kind of optimization problems is still very difficult to solve. To tackle this difficulty, a singular value decomposition method is proposed for solving the optimization problem. The details are discussed in the next section.

\section{Singular Value Decomposition Based Method}

We first study properties of an optimal solution of the unconstrained optimization problem, then an iterative algorithm is proposed for finding an optimal solution of the optimization problem.

Let an optimal solution of the unconstrained optimization problem be $\mathbf{U}_{c}^{\bullet}$ for $c=0,1, \cdots, C-1$. Define

$$
\nabla \widetilde{J}\left(\mathbf{U}_{c}\right) \equiv\left[\begin{array}{lll}
\frac{\partial}{\partial \mathbf{u}_{c, 0}} \widetilde{J}\left(\mathbf{U}_{c}\right) & \cdots & \frac{\partial}{\partial \mathbf{u}_{c, N-1}} \widetilde{J}\left(\mathbf{U}_{c}\right)
\end{array}\right]
$$


for $\quad c=0,1, \cdots, C-1 \quad$ Let $\left.\quad \nabla \widetilde{J}\left(\mathbf{U}_{c}^{\bullet}\right) \equiv \nabla \widetilde{J}\left(\mathbf{U}_{c}\right)\right|_{\mathbf{U}_{c}=\mathbf{U}_{c}^{\bullet}}$ for $c=0,1, \cdots, C-1$. Also, let $\mathbf{U}_{c, J}$ and $\mathbf{V}_{c, J}$ for $c=0,1, \cdots, C-1$ be Hermitian matrices and $\mathbf{D}_{c, J}$ for $c=0,1, \cdots, C-1$ be a diagonal matrix such that

$$
\nabla \widetilde{J}\left(\mathbf{U}_{c}^{\bullet}\right)=\mathbf{U}_{c, J} \mathbf{D}_{c, J} \mathbf{V}_{c, J}^{H} \text { for } c=0,1, \cdots, C-1 .
$$

Similarly, let an optimal matrix containing the optimal Lagrange multipliers be $\lambda_{c}^{\bullet}$ for $c=0,1, \cdots, C-1$. Let $\mathbf{U}_{c, \lambda}$ and $\mathbf{V}_{c, \lambda}$ for $c=0,1, \cdots, C-1$ be Hermitian matrices and $\mathbf{D}_{c, \lambda}$ for $c=0,1, \cdots, C-1$ be a diagonal matrix such that

$$
\lambda_{c}^{\bullet}+\lambda_{c}^{{ }^{H}}=\mathbf{U}_{c, \lambda} \mathbf{D}_{c, \lambda} \mathbf{V}_{c, \lambda}^{H} \text { for } c=0,1, \cdots, C-1 .
$$

Let

$$
\gamma_{c, n}=\frac{\operatorname{Re}\left(\mathbf{u}_{c, n}^{H} \hat{\mathbf{Y}}_{c} \mathbf{u}_{c, n}\right)}{\mathbf{u}_{c, n}^{H} \hat{\mathbf{X}}_{c} \mathbf{u}_{c, n}}
$$

for $c=0,1, \cdots, C-1$ and for $n=0,1, \cdots, N-1$. To solve the unconstrained optimization problem using conventional gradient descent approaches, we need to compute the gradient vectors of the optimization problem and an optimal matrix of the Lagrange multipliers. The following theorem characterizes the relationship among the gradient vectors, an optimal matrix of the Lagrange multipliers and an optimal solution.

Theorem 3

$$
\begin{aligned}
& \frac{\partial}{\partial \mathbf{u}_{c, n}} \hat{J}\left(\mathbf{U}_{c}, \boldsymbol{\lambda}_{c}\right) \\
& =\gamma_{c, n}^{2}\left(\left(\hat{\mathbf{X}}_{c}^{T}+\hat{\mathbf{X}}_{c}\right)-\frac{2}{\gamma_{c, n}}\left(\hat{\mathbf{Y}}_{c}^{T}+\hat{\mathbf{Y}}_{c}\right)\right) \mathbf{u}_{c, n}^{*}-\sum_{p=0}^{N-1}\left(\lambda_{c, p, n}+\lambda_{c, n, p}^{*}\right) \mathbf{u}_{c, p}^{*}
\end{aligned}
$$

for $c=0,1, \cdots, C-1$ and for $n=0,1, \cdots, N-1$. Also,

$$
\begin{gathered}
\mathbf{U}_{c, \lambda}=\mathbf{V}_{c, \lambda}=\mathbf{V}_{c, J} \text { for } c=0,1, \cdots, C-1, \\
\mathbf{D}_{c, \lambda}=\mathbf{D}_{c, J} \text { for } c=0,1, \cdots, C-1,
\end{gathered}
$$

and

$$
\mathbf{U}_{c}^{\bullet}=\mathbf{V}_{c, J} \mathbf{U}_{c, J}^{H} \text { for } c=0,1, \cdots, C-1 .
$$

By Theorem 3, we know that $\mathbf{U}_{c}^{\bullet}$ for $c=0,1, \cdots, C-1$ has to satisfy (22), where $\mathbf{U}_{c, J}$ and $\mathbf{V}_{c, J}$ for $c=0,1, \cdots, C-1$ are the singular value decomposition matrices of $\nabla \widetilde{J}\left(\mathbf{U}_{c}^{\bullet}\right)$ for $c=0,1, \cdots, C-1$. However, $\nabla \widetilde{J}\left(\mathbf{U}_{c}^{\bullet}\right)$ for $c=0,1, \cdots, C-1$ is also dependent on $\mathbf{U}_{c}^{\bullet}$ for $c=0,1, \cdots, C-1$. Hence, it is still difficult to find $\mathbf{U}_{c}^{\bullet}$ for $c=0,1, \cdots, C-1$. To tackle this difficulty, we first initialize an arbitrary Hermitian matrix $\mathbf{U}_{c}$ for $c=0,1, \cdots, C-1$. Then, we compute $\nabla \widetilde{J}\left(\mathbf{U}_{c}\right)$ for $c=0,1, \cdots, C-1$ and the corresponding singular value decomposition matrices $\mathbf{U}_{c, J}, \quad \mathbf{D}_{c, J}$ and $\mathbf{V}_{c, J}$ for $c=0,1, \cdots, C-1$. By substituting these matrices to (20)-(22), a new Hermitian matrix $\mathbf{U}_{c}$ for $c=0,1, \cdots, C-1$ is obtained. The above procedures are iterated until the algorithm converges or the number of iterations has exceeded the prescribed value. Finally, take the obtained solution as a locally optimal solution of the optimization problem. The algorithm is summarized as follows:

\section{Algorithm 1}

Step 1: Initialize an arbitrary Hermitian matrix $\mathbf{U}_{\mathrm{c}, 0}$ for $c=0,1, \cdots, C-1$, set an iteration index $k=0$, define an acceptable stopping criterion be $\varepsilon$ and the maximum number of iterations be $T$.

Step 2: Compute $\nabla \widetilde{J}\left(\mathbf{U}_{c, k}\right)$ for $c=0,1, \cdots, C-1$.

Step 3: Compute the singular value decomposition matrices of $\nabla \widetilde{J}\left(\mathbf{U}_{c, k}\right)$ and denote as $\widetilde{\mathbf{U}}_{c, k, J}, \widetilde{\mathbf{D}}_{c, k, J}$ and $\tilde{\mathbf{V}}_{c, k, J}$ for $c=0,1, \cdots, C-1$.

Step 4: Compute a new Hermitian matrix $\widetilde{\mathbf{U}}_{c, k+1}=\widetilde{\mathbf{V}}_{c, k, J} \widetilde{\mathbf{U}}_{c, k, J}^{H}$ for $c=0,1, \cdots, C-1$.

Step 5: If $\left|\widetilde{J}\left(\mathbf{U}_{c, k-1}\right)-\widetilde{J}\left(\mathbf{U}_{c, k}\right)\right|>\varepsilon$ and $k<T$, where $c=0,1, \cdots, C-1$, then increment the value of $k$ and go to Step 2. Otherwise, take $\widetilde{\mathbf{U}}_{c, k+1}$ for $c=0,1, \cdots, C-1$ as an optimal solution.

It is worth noting that the computational complexity of the algorithm is not an issue because the number of iterations is bounded by $T$. Also, if the algorithm converges, the obtained solution is guaranteed to be a local optimal solution of the optimization problem. This is because Theorem 3 is satisfied in this scenario. Although theoretically that i) the algorithm may converge at the iteration index greater than $T$, or ii) the algorithm may converge with the difference between two consecutive cost values smaller than $\varepsilon, T$ and $\varepsilon$ can be set at a very large value and a very small value, respectively, to guarantee the algorithm does not terminate before it converges. However, increasing the value of $T$ and decreasing the value $\varepsilon$ will increase the computational complexity of the algorithm. Hence, there is a tradeoff between the computational complexity and the accuracy of the obtained solution. Moreover, although theoretically that may be the case that $\left|\widetilde{J}\left(\mathbf{U}_{c, k-1}\right)-\widetilde{J}\left(\mathbf{U}_{c, k}\right)\right|$ for $c=0,1, \cdots, C-1$ may not converge due to the possibility of the exhibition of limit cycle, chaotic or divergent behaviours of $\widetilde{J}\left(\mathbf{U}_{c, k}\right)$ for $c=0,1, \cdots, C-1$, numerical computer simulations based on an intensive set of arbitrary $\mathbf{U}_{\mathrm{c}, 0}$ for $c=0,1, \cdots, C-1$ show that this is not the case.

In order to kick out the obtained locally optimal solution and obtain a better locally optimal solution, an intensive set of arbitrary $\mathbf{U}_{\mathrm{c}, 0}$ for $c=0,1, \cdots, C-1$ can be generated and pick up the solution corresponding to the minimum objective value. 
Similarly, this will increase the computational complexity of the algorithm. Hence, there is also a tradeoff between the computational complexity and the accuracy of the obtained solution.

After $\mathbf{U}_{c}$ and $\mathbf{f}_{c}$ for $c=0,1, \cdots, C-1$ are obtained from the above, the classification can be performed based on the following algorithm:

Algorithm 2

Step 1: For a test image with the feature vector $\mathbf{z}$, compute

$$
g_{c}(\mathbf{z}) \equiv\left\|\mathbf{U}_{c}^{H} \mathbf{F}_{c} \mathbf{U}_{c} \mathbf{z}-\mathbf{y}_{c}\right\|^{2} \text { for } c=0,1, \cdots, C-1 .
$$

Step 2: Assign $\mathbf{z}$ to the class $c^{*}$, where

$$
g_{c^{*}}(\mathbf{z})=\min \left(g_{0}(\mathbf{z}), \cdots g_{C-1}(\mathbf{z})\right) \text {. }
$$

The above algorithm is basically to assign $\mathbf{z}$ to the class where the Euclidean 2-norm of the difference between the processed feature vector and the represented feature vector of the class is minimum.

\section{Numerical COMPUter SimUlation Results}

In this work, we first divide an image of size 576 x 768 pixels into 32 x 32 pixel sub-images. Subsequently, feature extraction will be applied to these sub-images to characterize each image for anomality/normlaity detection.

\section{A. Features}

In this work, key features that are capable of representing a normal and abnormal image (As illustrated in Figures 1 and 2 , respectively) must be obtained. Texture features are an important characteristic in retina images due to the way in which they can appear. For example, background sub-images will have a smooth texture whereas clusters of exudates or hemorrhages (Figure 2) will appear to have a rough texture. On the other hand, blood vessels (Figure 1) will appear to have a random, periodic texture. Hence, texture characteristics are considered an important feature in this work. The list of texture features and edge features extracted from the sub-images are illustrated in Table I.

\section{TABLE I: List of FeATURES USED}

\begin{tabular}{|l|l|}
\hline \multicolumn{1}{|c|}{ Feature } & \multicolumn{1}{c|}{ Purpose } \\
\hline Standard Deviation & $\begin{array}{l}\text { Measure the variance within the sub- } \\
\text { image }\end{array}$ \\
\hline Edge Response & $\begin{array}{l}\text { Determines total sum of vertical and } \\
\text { horizontal response from Sobel } \\
\text { gradient operators. }\end{array}$ \\
\hline Smoothness & $\begin{array}{l}\text { Measures the relative smoothness of } \\
\text { the intensity in a region }\end{array}$ \\
\hline Uniformity & Measures uniformity of the region \\
\hline Entropy & $\begin{array}{l}\text { Measures randomness within the } \\
\text { region }\end{array}$ \\
\hline
\end{tabular}

All of these features extracted in each sub-image are then reorganized into a single vector to represent one image. Naturally, as each 576 by 768 pixels image have a total of 352 sub-images, the feature vectors will have too high dimensions for effective classification. Principal component analysis was used to reduce this into more manageable dimensions. In this work, 100 components were used as our initial analysis demonstratd they gave the best results.

\section{B. Data Sets}

A total 195 images were used for both training and testing of the algorithm. Among these data, 50 normal images and 50 abnormal images were used for training. The rest of the 95 images were used for testing.

\section{Experimental Performance}

The results were evaluated based on the total number of images correctly identified. In total, we have 70 normal test images and 25 abnormal test images. Table II represents the number of correctly identified images. Based on the results, the number of correctly identified image gives a total accuracy of $81 \%$, with $100 \%$ sensitivity and $74.3 \%$ specificity.

\section{TABLE II: NUMBER OF CORRECTLY IDENTIFIED}

\begin{tabular}{|l|l|}
\hline \multicolumn{1}{|c|}{ Type } & Correctly Identified \\
\hline Normal & 52 out of 70 \\
\hline Abnormal & 25 out of 25 \\
\hline
\end{tabular}

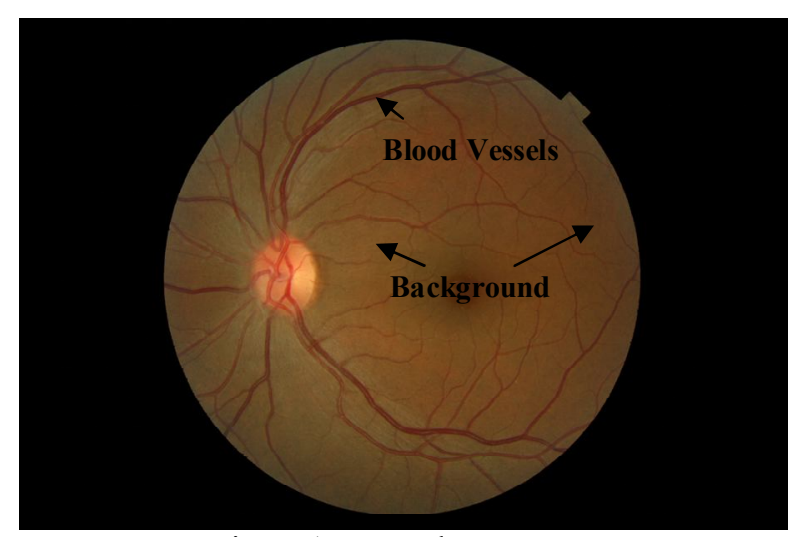

Figure 1. Normal Image.

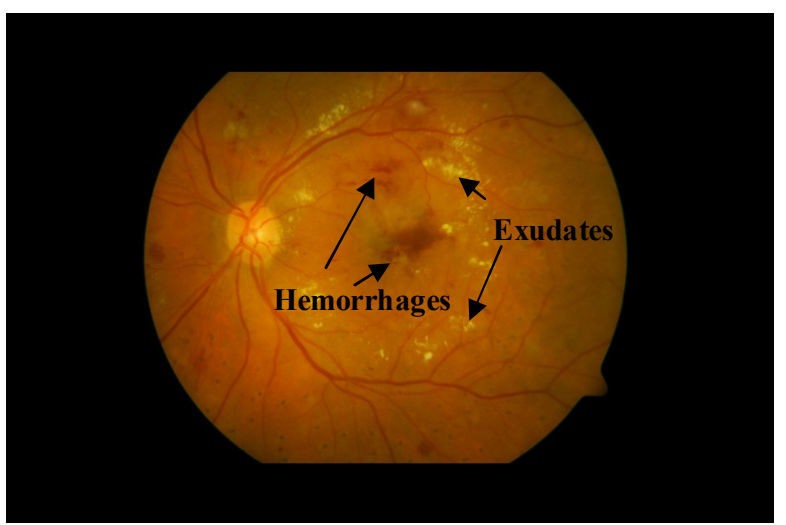

Figure 2. Abnormal Image. 


\section{CONCLUSION}

This paper has derived a novel methodology for the simultaneous and optimal designs of both the Hermitian transforms and the masks for reducing the intraclass separations of feature vectors for anomaly detection of diabetic retinopathy images. The optimal masks are expressed in terms of the optimal Hermitian transforms. The optimal designs of the Hermitian transforms are actually optimization problems with highly nonlinear objective functions subject to complex valued quadratic Hermitian constraints. A singular value decomposition approach is proposed for deriving a condition on the optimal solutions of the optimization problems and an iterative approach is proposed for solving the optimization problems. In this experiment, we obtained very high sensitivity, $100 \%$, that is, the system detected all the disease cases. This also means that among all the images detected as normal ones, they were indeed normal. There were 18 images which the system considered as abnormal and in fact they were normal, this is acceptable in practice if the system serves as a screening tool, as such images will be checked again by medics. This method may fail in those cases when there are only very subtle changes in those with early retinopathy. However, the positive outcome of this work demonstrates its potentials of being integrated into our other ongoing work carried out at the University of Surrey which focuses more on the detailed detections [5]. If such performance is maintained when testing on very large-scale images across diverse patient populations, this may indicate the system potentially is able to filter out most of the normal images without any false negative.

\section{ACKNOWLEDGEMENT}

This work is in collaboration with the Reading Centre, Department of Research and Development, NIHR Biomedical Research Centre for Ophthalmology, at Moorfields Eye
Hospital NHS Foundation Trust and UCL Institute of Ophthalmology, United Kingdom.

\section{REFERENCES}

[1] A. D. Fleming, K. A. Goatman, S. Philip, J. A. Olson and P. F. Sharp, "Automatic detection of retinal anatomy to assist diabetic retinopathy screening," Physics in Medicine Biology, vol. 52, no. 2, pp. 331-45, 2007.

[2] A. Osareh, M. Mirmehdi, B. Thomas and R. Markham, "Automated identification of diabetic retinal exudates in digital color images," British Journal of Ophthalmology, pp. 1220-1223, 2003.

[3] J. M. Shivaram, R. Patil and H. S. Aravind, "Automated detection and quantification of haemorrhages in diabetic retinopathy images using image arithmetic and mathematical morphology methods," International Journal of Recent Trends in Engineering, vol. 2, no. 6, 2009.

[4] C. Sinthanayothin, J. F. Boyce, H. L. Cook and T. H. Williamson, "Automated localisation of the optic disc, fovea, retinal blood vessels from digital colour fundus images," British Journal of Ophthalmology, vol. 93, pp. 902-910, 1999.

[5] H. L. Tang, T. Peto, J. Goh, Y. Jin and C. Chuluunkhuu, "Filtering normal diabetic retinopathy images through eveloluionary computation," European Journal of Opthamology, vol. 21, no. 3, pp. 347-348, 2011

[6] C. Y. F. Ho, B. W. K. Ling, H. K. Lam and M. H. U. Nasir, "Gloabl convergence and limit cycle behavior of weights of percepron," IEEE Transactions on Neural Networks, vol. 19, no. 6, pp. 938-947, 2008.

[7] M. Ezoji and K. Faez, "Use of matrix polar decomposition for illumination-tolerant face recognition in discrete cosine transform domain," IET Image Processing, vol. 5, no. 1, pp. 25-35, 2011.

[8] S. R. Subramaniam, B. W. K. Ling and A. Georgakis, "Filtering in rotated time-frequency domains with unknown noise statistics," IEEE Transactions on Signal Processing, vol. 60, no. 1, pp. 489-493, 2012.

[9] C. Y. F. Ho, B. W. K. Ling, L. Benmesbah, T. C. W. Kok, W. C. Siu and K. L. Teo, "Two-channel linear phase FIR QMF bank minimiax design via global nonconvex optimization programming," IEEE Transactions on Signal Processing, vol. 58, no. 8, pp. 4436-4441, 2010. 